\title{
ESTRATEGIAS DE AFRONTAMIENTO EN VÍCTIMAS DESPLAZADAS DEL CORREGIMIENTO DE EL SALADO, BOLÍVAR
}

\author{
Darlyn Danessa Anaya Mercado ${ }^{1}$ \\ Fundación Semillas de Vida para Colombia \\ Viviana Patricia Romero Pérez ${ }^{2}$ \\ Fundación para el Desarrollo Social Comunitario la Luz
}

\section{Resumen}

El objetivo de la presente investigación es describir las estrategias de afrontamiento desarrolladas por dos víctimas del desplazamiento forzado del corregimiento El Salado, ubicado en el municipio El Carmen de Bolívar, departamento de Bolívar, Colombia. Para lograr el objetivo se recurrió a la técnica de la entrevista, aplicada a las víctimas, fueron entrevistados sus familiares y a dos personas expertas para corroborar la información y realizar una triangulación de los datos obtenidos. Para el análisis de las entrevistas se utilizó el software ATLAS TI 7.5.12, ya que es un programa de carácter cualitativo que facilita la organización y seguidamente el análisis de la información. Los resultados más relevantes develan que las dos víctimas del desplazamiento forzado han desarrollado estrategias de afrontamiento cognitivas, entre las cuales están la preocupación, planificación y reevaluación positiva; además, hacen uso de las estrategias de tipo afectivo como el apoyo social, y emplean estrategias conductuales como la falta de afrontamiento para resistir esta experiencia traumática. Finalmente, cabe destacar que en la investigación se evidenció una nueva estrategia la cual no se encontraba dentro de las categorías establecidas, y se denominó estrategia de afrontamiento basada en la $\mathrm{fe}$, dado que, las personas entrevistadas manifestaron que afrontaron la situación creyendo en Dios.

1 Psicóloga. Fundación semillas de vida para Colombia. Correo: dadamer16@gmail.com.

2 Psicóloga. Fundación para el desarrollo social comunitario la luz. Correo: romero.viviana2803@gmail.com. 
Estrategias de afrontamiento en víctimas desplazadas del corregimiento del Salado, Solívar. Un estudio de caso

Palabras clave: estrategias, desplazamiento, violencia, afrontamiento, victimas.

\section{Abstract}

The objective of this research is to describe the coping strategies developed by two victims of forced displacement, of the corregimiento El Salado, Bolívar. To achieve the objectives an interview was conducted with each participant, also interviews with their relatives, followed by an interview with two experts to corroborate the information and make triangulation of the data obtained. For the analysis of the interviews was used the software ATLAS TI 7.5.12, because it is a qualitative program that facilitates the organization and then the analysis of the information. The results show that the two victims of forced displacement have developed cognitive coping strategies, among which are concern, planning and positive reassessment; In addition, they make use of coping strategies of an affective type such as social support and use behavioral coping strategies such as lack of coping to face this traumatic experience. Finally, it should be noted that the research found a new strategy which was not within the established categories, this takes as a coping strategy based on faith, given that the people interviewed said they faced the situation believing in God.

Keywords: strategies, displacement, violence, coping, victims. 


\section{Introducción}

El Corregimiento El Salado fue fundado en el año 1812, pertenece al municipio del Carmen Bolívar, ubicado en los Montes de María. Para la década de los noventa su principal actividad económica era la producción de tabaco y el cultivo de maíz, ajonjolí, ahuyama y ñame, así como también la ganadería. Esto debido a que se encontraba dotado de zonas acuíferas que facilitaban la siembra de dichos productos. Con la incursión de la producción de tabacos, grandes empresas tabacaleras se fijaron en esta tierra y se instalaron. A partir de la instalación de estas grandes empresas aumentó la economía del corregimiento y la calidad de vida de sus habitantes; hubo más empleos -llegó a tener 33 tiendas-, almacenes, depósitos y droguerías (Sánchez, 2009).

En el año 2000, el corregimiento El Salado inicia una transición que marcaría su historia, pus ocurrieron las primeras masacres por parte de los grupos paramilitares comandados en ese entonces por Rodrigo Tovar Pupo —alias "Jorge 40" - Salvatore Mancuso y John Henao —alias "H2"-, quienes eran delegados de Carlos Castaño. Para los días del 16 al 21 de febrero del 2000 se llevaron a cabo 60 asesinatos y dos violaciones por parte de este grupo al margen de la ley, dejando como resultado 52 hombres y 8 mujeres fallecidos, entre los cuales podemos destacar 3 menores de 18 años, 12 que oscilaban entre los 18 y 25 años, 10 entre los 26-35, 23 entre los 36-55 y 10 adultos mayores que arrojaría un total de 60 víctimas mortales; esto desencadenó un desplazamiento forzado de 4000 personas aproximadamente, llevándolos a municipios como el Carmen de Bolívar y ciudades aledañas como Sincelejo, Cartagena y Barranquilla (Sánchez, 2009).

Haciendo referencia a dicho tema, Rodríguez (2006, p. 260) indica: "Colombia es el tercer país con mayor índice de desplazamiento en el mundo después de Angola y Sudán". Esto ha llevado a tomar la iniciativa de conocer y ser conscientes de la situación que vive el país, en la que todos estamos implicados directa o indirectamente. Por lo que Cervellin y Uribe (citados por Rodríguez, 2006, p. 260) plantean que este hecho comienza a tener un auge de manera oficial en el año 1995, cuando se publica el primer estudio de esta problemática, periodo en que los investigadores enfatizan en este acontecimiento, buscando explicar, comprender y realizar 
Estrategias de afrontamiento en víctimas desplazadas del corregimiento del Salado, Solívar. Un estudio de caso

propuestas de intervención con la finalidad de atender las consecuencias a nivel psíquico y social creadas por la violencia.

Así mismo, la Organización Internacional para las Migraciones (OIM) y la Pontificia Universidad Javeriana, han manifestado que los factores causantes de este fenómeno son complejos, entre ellos: la fuerza que han tomado los grupos al margen de la ley, la toma de territorios para obtener una mayor expansión. Esto a través de actividades ilegales como la extorsión, el secuestro y el expendio de drogas. Lo cual trae condiciones de inseguridad, vulnerabilidad en civiles y atentados contra sus vidas, pues provocan temor y los obligan a desplazarse y a empezar de nuevo en otros sitios lejos de sus viviendas y bienes materiales. Por lo tanto, esto se convierte en una situación de peligro para la sociedad y a su vez en acontecimientos de tristeza y dolor para los afectados directamente (Rodríguez, 2006).

Estas situaciones están relacionadas con distintas formas de violencia, con el fin de imponer un control social por parte de los grupos armados, tales como: las torturas, amenazas directas a la población civil, masacres a grupos, es decir, a la vista de todos los habitantes. Por lo que también se originan cambios significativos en las relaciones familiares y sociales (Organización Internacional para las Migraciones -OIM y Pontificia Universidad Javeriana, citado por Rodríguez, 2006).

A raíz de esto, los supervivientes que se desplazan inician un nuevo proceso de adaptación lejos de sus costumbres y estilos de vida, conllevándolos a buscar nuevas estrategias de afrontamiento y de superación ante dicha situación.

El afrontamiento consiste en un proceso que integra diversas estrategias: psicológicas y comportamentales que permite enfrentarse a eventos estresores de la mejor forma posible, para tratar de reducir o controlar las amenazas internas o externas que son negativas para las personas (Lazarus y Folkman, 1986, pág. 164 citado por Vázquez, Crespo y Ring, 2000). Ante esto, se tiene en cuenta que el uso de estas estrategias puede estar determinado por el tipo del factor estresante y las condiciones en que se origina (Forsythe y Compas 1987); es decir, pueden ser estrategias centradas en la resolución del problema o estrategias centradas en las emociones (Vázquez, Crespo y Ring, 2000). 
Tomando como referencia la validez y confiabilidad de esta tipología propuesta en primera instancia por estos autores, estudios más recientes han presentado la existencia de otros estilos de afrontamiento. Una de esas investigaciones hace énfasis en la escala de modos de Afrontamiento Ways of Coping, WOC_-, (Folkman y Lazarus, 1988), un instrumento diseñado para evaluar de forma holística diversos pensamientos y conductas que se emplean para controlar un suceso amenazante. Se basa en ocho estrategias distintas que son: confrontación, planificación, distanciamiento, autocontrol, aceptación de responsabilidad, evitación, reevaluación positiva, estas 7 se basan en la regulación emocional del individuo, mientras que la búsqueda de apoyo social, se centra en las dos áreas.

Acerca de esta temática, para el año 2004 se publica un artículo en el cual se analizaron los cambios y resiliencia de las familias desplazadas por la violencia en Bogotá. Basándose principalmente en identificar la capacidad que tuvieron estas personas para afrontar distintas situaciones desfavorables, que trajeron consigo mucho dolor y tristeza para sus vidas - como resultado de las atrocidades realizadas por los responsables del conflicto armado-. Según este estudio las transformaciones en la estructura familiar se evidencian con mayor exactitud entre los momentos del desplazamiento y durante los primeros meses de su establecimiento en un lugar específico, para este caso en Bogotá, distrito capital. Pero lo más significativo que descubrieron, lo cual es de relevancia para la presente investigación fueron las estrategias de afrontamiento que desarrollaron las víctimas para perseverar en todo momento y lugar; buscando propósitos para favorecer la unión y fraternidad de la familia. Tales herramientas son el buen humor para su comunicación, es decir, "a través de la risa las mujeres logran un determinado control de la difícil situación”, resolver problemas conjuntamente, generosidad con el otro y construcción de nuevas redes de apoyo (Cyrulnik, así como es citado por Gonzales, 2004, p. 128).

En el año 2016 se publica una investigación realizada con adultos expuestos al desplazamiento forzado por el conflicto armado en Colombia. Centrada en determinar las afectaciones psicológicas, estrategias de afrontamiento y niveles de resiliencia desarrollados ante este suceso. Se encontró que el alcoholismo, las alteraciones en el estado de ánimo, la sensación o percepción de que alguien le hace daño y síntomas del estrés postraumáticos, son afectaciones psicológicas ocasionadas al estar 
Estrategias de afrontamiento en víctimas desplazadas del corregimiento del Salado, Solívar. Un estudio de caso

expuestos al conflicto armado, utilizando las estrategias de afrontamiento como la religión y esperar que las cosas se resuelvan solas y estableciendo un nivel de resiliencia moderado. (Hewitt, et al., 2016).

Las ideas precedentes sirven de marco a esta investigación cuyo objetivo es describir las estrategias de afrontamiento que han desarrollado dos víctimas del desplazamiento forzado del corregimiento El Salado, Bolívar. Para lo cual se han considerado las argumentaciones teóricas de autores como: Folkman y Lazarus (1988); Moos y Moos (1988); Carver, Scheier y Weintraub, (1989); Páez (1993); Galán y Perona (2001), Martin (2004), quienes plantean que existen tres tipos generales de estrategias de afrontamiento, el cognitivo, el afectivo y el conductual. A continuación, se presenta un inventario de estrategias que aluden a los procesos utilizados en cada contexto:

\section{Afrontamiento cognitivo:}

- Aceptación: implica reconocer, respetar y valorar a la situación y a sí mismo.

- Anticipación: Se refiere a la anticipación que se hace frente a los problemas con el fin de considerar las posibles reacciones emocionales y respuestas o soluciones a estos.

- Autoanálisis: consiste en el proceso de reflexionar sobre las propias ideas, sentimientos y conductas, reconociendo la autorresponsabilidad en el origen, sostenimiento del problema a fin de actuar acorde a esta reflexión.

- Humor: afrontar la situación angustiante a través del humor con aspectos irónicos y cómicos.

- Planificación: estudiar la problemática, y analizar las distintas opciones con el fin de desarrollar técnicas para darle una solución.

- Poder mental: se basa en técnicas metafísicas, centrados en el poder del pensamiento, afirmaciones y peticiones.

- Preocupación: hace referencia a una preocupación improductiva por el futuro, la cual se relaciona con afectividades negativas como la angustia, la infelicidad y el pesimismo. 
- Reestructuración mental: indica proyectarse a una transformación, de manera positiva, de la personalidad.

- Reevaluación positiva: hacer un cambio ante el significado de la situación problema, resaltando aspectos positivos que este tenga, expresando optimismo y una parte positiva de la situación.

Afrontamiento afectivo: en esta categoría se pueden mencionar algunas estrategias como las que seguidamente son descritas.

- Agresión pasiva: son aquellas manifestaciones agresivas hacia las demás personas ya sea de manera directa y no asertiva. Esto, como respuesta a exigencias de desempeño independiente, o la ausencia de agrado de deseos de independencia.

- Hostilidad o resentimiento latente hacia los demás: esta agresión pasiva se da como mecanismo de defensa, dado que la agresividad se ha desligado de su motivo, pudiendo o no existir una conciencia del sentimiento.

- Aislamiento social: indica una retirada de interacción social con familiares, compañeros o amigos, bien sea por querer afrontar la problemática solo, o un escape del resto de personas por reservar, o las dos cosas.

- Apoyo social: implica asistir a otras personas cercanas como amigos o familiares, organizaciones, grupos, líderes espirituales o profesionales, con el fin de buscar ayuda material como dinero, compartir la experiencia con los demás, información o apoyo emocional y espiritual.

- Autocontrol: realizar un esfuerzo por dominar y disimular los sentimientos y respuestas afectivas propias.

- Catarsis: se trata de soltar las emociones, expresar lo que se siente y esos estados emocionales intensos para afrontar eficazmente las situaciones difíciles.

- Escape/evitación: alejarse de la problemática, no pensar en eso y pensar en otras cosas, con el propósito de impedir una afectación 
Estrategias de afrontamiento en víctimas desplazadas del corregimiento del Salado, Solívar. Un estudio de caso

emocional, utilizando pensamientos irreales, improductivos o desiderativos y realizando actividades que lo distraigan.

- Supresión: se basa en eludir intencionalmente, ideas, recuerdos, sentimientos o experiencias molestosos.

Afrontamiento conductual: Seguidamente se mencionan las siguientes estrategias:

- Afrontamiento directo: enfrentar directamente la situación por medio de actitudes directas, agresivas o altamente arriesgadas.

- Autoafirmación: implica expresar los pensamientos o sentimientos directamente, sin agresividad ni manipulación, es decir, de manera asertiva.

- Falta de afrontamiento: incapacidad del sujeto para afrontar los problemas, debido a la falta de dominio sobre sí mismo o al considerarlo irresoluble, desarrollando síntomas psicosomáticos y actitudes depresivas.

- Postergación: la persona espera que se dé la ocasión indicada para actuar, posponiendo todas las actividades hasta tener una mayor y mejor información del suceso.

Ahora bien, cabe destacar que esta investigación es de gran valor para indagar las estrategias de afrontamiento que fueron utilizadas por personas víctimas del conflicto armado, por lo que en este caso serán dos personas nativas del corregimiento El Salado, Bolívar, quienes suministren la información y sean el centro de atención del estudio. Pues, aunque se haya investigado sobre lo sucedido en este lugar, es decir, las masacres, quiénes la cometieron y cómo lo hicieron, aún existen vacíos de información que permitirían comprender desde una perspectiva abordada individualmente lo que aconteció con esas víctimas después de su desplazamiento a otras ciudades. Por lo tanto, al realizar este estudio se logrará ampliar teóricamente esta historia vivida en Colombia por más de 50 años.

Siguiendo esta línea de pensamiento, es importante indagar estos temas, pues, debemos interesarnos en lo que ha sucedido en nuestro país, y en este caso, específicamente en nuestra región, la cual ha sido una de las más azotadas por el conflicto armado. De igual forma, los conocimientos obtenidos serán de gran ayuda a los profesionales que 
brindan acompañamientos psicosociales en los centros de apoyo a víctimas, pues esto facilitaría los procesos de atención.

Por otro lado, es importante señalar que existe un estudio de víctimas de desplazamiento realizado en la Corporación Universitaria del Caribe CECAR realizado por Álvarez, Velilla \& Amell (2018), en el cual se tienen en cuenta las estrategias de afrontamiento a partir del desplazamiento forzado que vivieron ciertas personas por el conflicto armado, en esta investigación se consideró relevante conocer este aspecto y lo que ha acontecido en los ámbitos familiar, social y económico, luego de lo vivido por dichas víctimas. Conocer esta problemática es significativo, puesto que, CECAR tiene entre su misión la "responsabilidad social" como uno de sus objetivos banderas, y realizar investigaciones de esta naturaleza, ayuda a desarrollar la proyección social que es tan importante, no solo para la institución, sino también, para el Programa de Psicología, acreditado el año 2018. Se espera, que esta investigación cree una nueva perspectiva con respecto a las personas que utilizan las estrategias de afrontamiento, lpara superar este tipo de situaciones que ha hecho que ellos transformen su estilo de vida cotidiano, puesto que dejan sus costumbres, su tierra y sus bienes materiales para comenzar una vida, quizás diferente a la que llevaban.

\section{Metodología}

\section{Tipo de investigación}

La presente investigación es descriptiva, insertada en un enfoque de tipo cualitativo: se describe un fenómeno considerando la perspectiva del participante; la información aportada por él según su vivencia es clave, pues permite comprender la situación objeto de indagación mediante el análisis y significado de su discurso.

\section{Diseño de Investigación}

El diseño de la investigación se encuentra centrado en el estudio de caso, el cual según Monje (2011) es el estudio propio de un caso o de pocos casos con relación a una situación en común, que busca conocer de manera holística problemas generales 
Este tipo de estudio es adecuado para aplicarse a circunstancias en la que se quieren investigar elementos tales como: las características esenciales de los individuos y la situación que están viviendo personas pertenecientes a algún tipo de población o entidades. Ejemplo: estudio de la conducta social de dos jóvenes adictos al alcohol.

Monje (2011), plantea que las etapas que se llevan a cabo para la realización de un estudio de caso son:

1. Formular los objetivos del estudio, especificando cuál es el caso y qué características, elementos y procesos se van a observar.

2. Explicar cómo se escogió el caso a estudiar y qué técnicas de observación se van a llevar a cabo en el desarrollo de este.

3. Recolectar la información.

4. Organizar la información estableciendo una forma coherente que componga el caso que se estudia.

5. Realizar los resultados y la discusión pertinente tomando como base los objetivos estipulados.

\section{Procedimiento del estudio}

A continuación se expone el procedimiento implementado en la investigación:

1-Se planteó la razón y los objetivos para realizar la indagación; se establecieron las técnicas y procesos para obtener la información.

2- Se realizó una revisión documental para explorar la información acerca de la temática y la población, para luego, analizarla y seleccionar las fuentes confiables que servirían de soporte para la investigación.

3- Se diseñó una entrevista semiestructurada, la cual se sometió a una prueba piloto aplicándola a una persona que también había sido desplazada, pero que no sería parte de la muestra a investigar. Esta prueba preliminar permitió afinar el instrumento adecuando el lenguaje, la temática y la estructura, para someterlo a un proceso de validación, mediante el juicio de expertos en la temática, obtenida la valoración de los jueces, se elaboró el instrumento definitivo. 
4- Se procedió a seleccionar entre la población de la localidad a las personas que estuvieran dispuestas a participar y aportar información conveniente para el proyecto, una vez dado este paso se estableció un contacto cercano para explicarles en qué consistía el estudio y acordar fechas de la entrevista.

5- Mediante un documento que exponía los objetivos del estudio, se obtuvo el consentimiento informado de los participantes y fue solicitado su permiso para publicar bajo anonimato la información que suministraran.

6- Se realizó la entrevista a la muestra seleccionada (dos personas), con el fin de recolectar la información requerida para el estudio. Se recurrió al muestreo teórico definido por Glaser $\&$ Strauss (1967), en el cual se eligen los primeros sujetos o unidades de análisis y se analizan los datos por ellos aportados, seguidamente se desarrollan conceptos así como categorías que se usan para elegir a otros grupos de sujetos o unidades de análisis, y así sucesivamente hasta que se saturen las categorías, de modo que emerja la teoría.

7-A partir de la información obtenida se diseñó otra entrevista que fue aplicada a un familiar de cada participante, es decir, a dos personas más, y de este modo confirmar la información anteriormente recolectada.

8- Se procedió a configurar una nueva entrevista la cual fue aplicada a dos personas expertas en el tema de estrategias de afrontamiento, conflicto armado colombiano y la violencia vivida por los habitantes El Salado con el fin de corroborar la información de los casos estudiados.

9- Recolectada toda la información se realizó análisis exhaustivo del discurso, ello permitió el desarrollo de conceptos y categorías.

10- Se procedió a la discusión de los datos, a su triangulación y elaboración de las conclusiones.

\section{Participantes}

Participaron en el estudio dos personas desplazadas del corregimiento El Salado, víctimas de la masacre del 2000. Según el informe elaborado por el Instituto Colombiano de Bienestar Familiar - ICBF Centro Zonal Carmen de Bolívar en 20023 el porcentaje de la población desplazada se distribuyó

3 Diagnóstico Comunitario Comunidad de EL Salado (Bolívar) año 2002", informe ela- 
Estrategias de afrontamiento en víctimas desplazadas del corregimiento del Salado,

de la siguiente manera: de todas las familias, 55.2\% se estableció en Carmen de Bolívar; 19.7\% se trasladó a Barranquilla, 15.7\% se ubicó en la ciudad de Sincelejo y 9.21\% se ubicó en Cartagena (Resolución defensorial regional $n^{\circ}$. 008, 2002). Sin embargo, actualmente no existe un reporte con cifras exactas de las víctimas del conflicto armado El Salado que estén residentes en otras ciudades.

Debido a esto, se realizó un muestreo por conveniencia, es decir, de manera intencional fueron seleccionadas dos víctimas del desplazamiento forzado del corregimiento El Salado, Bolívar, en el año 2000, residentes de la ciudad de Sincelejo, fueron las personas más cercanas y asequibles para la realizar la investigación. La muestra quedó conformada por dos hombres mayores de edad (40 y 55 años respectivamente). Asimismo, se escogió un familiar de cada uno de los sujetos de estudio, es decir, dos personas más, las cuales tenían conocimiento de la experiencia por la que había pasado el participante seleccionado en el caso de estudio.

Finalmente, se eligieron dos personas expertas en la temática a tratar, estas han trabajado con víctimas del desplazamiento forzado desde su profesión —un trabajador social y un psicólogo-. Conocieron las experiencias y las formas cómo estas personas han afrontado la situación del desplazamiento, con el fin de tener una mayor información acerca de lo que sucede en la vida de los sujetos de estudio y así mismo, lograr una mayor fundamentación en esta investigación.

\section{Contextualización}

Esta investigación se llevó a cabo desde el año 2017 hasta el año 2018, comenzando con su planteamiento en las aulas de la sede principal de la Corporación Universitaria del Caribe - CECAR con nuestros docentes de cátedras investigativas. Se continúa la ejecución de la investigación en el barrio Pioneros de la ciudad de Sincelejo, lugar donde viven los participantes, dado a que las entrevistas se realizaron en sus viviendas. De esto, logramos observar que ellos vivían con su núcleo familiar y se desarrolló la entrevista de una manera adecuada y fluida. Por otra parte, las entrevistas con las personas expertas fueron en el centro de familia de

borado por el ICBF Centro Zonal Carmen de Bolívar en Julio de 2002. 
CECAR, se hizo el análisis de la información elaborado en la sede principal de CECAR.

\section{Instrumento}

Para la recolección de la información de la investigación se hizo una revisión bibliográfica de las categorías a trabajar en el estudio, a fin de obtener información suficiente de lo que se quiere estudiar.

Asimismo, se utilizó una entrevista semiestructurada, la cual consiste en tener una guía de preguntas claves con relación a la temática que se quiere investigar, no hay una secuencia en el cuestionario, ya que todo depende de las respuestas recibidas por el entrevistado. Este tipo de técnica se debe realizar en un ambiente cordial y empático (Martínez 2011). Este tipo de entrevista se aplicó a los dos sujetos seleccionados, y de este modo, se consiguió información desde lo vivenciado por ellos, de igual manera, como ya se mencionó, también se aplicó a un familiar de estos, con el fin de corroborar la información suministrada, adicionalmente a dos personas expertas en la temática del desplazamiento forzado en el El Salado, Bolívar.

\section{Análisis de información}

Para el análisis de los datos obtenidos se utilizó la técnica de triangulación de datos dado que se utilizan diferentes fuentes de datos en la investigación. La triangulación es una forma de comprobar y contrastar la información que se consigue en los distintos momentos en que se han utilizado los métodos (Patton, 2002), los cuales son: la revisión bibliográfica, las entrevistas semiestructuradas a los dos partícipes del estudio de caso, a un familiar de cada uno de ellos a dos personas expertas en el tema, sumando en total seis entrevistados. En cada una de las entrevistas realizadas se hizo un análisis de los datos, para así obtener temáticas generales tratadas en el discurso de los entrevistados. Posteriormente, se hace uso del software de análisis cualitativo ATLAS ti 7.5.12, dado que este nos permite organizar la información importante, establecer categorías, códigos y redes que faciliten el análisis de los datos (Ávila, 2014). 
Estrategias de afrontamiento en víctimas desplazadas del corregimiento del Salado,

\section{Resultados}

A continuación, se presentan las tablas en las que se presentan los hallazgos, el contenido de las mismas se completa breve descripción basada en la teoría expuesta, avanzando así hacia el logro de los objetivos planteados en esta investigación.

\section{Tabla 1}

Categorías de análisis de acuerdo a los referentes teóricos y los datos obtenidos.

\begin{tabular}{|c|c|c|c|}
\hline Objetivos & Categoría & subcategorías & Propiedades \\
\hline \multirow{3}{*}{$\begin{array}{l}\text { Objetivo General } \\
\text { Describir las } \\
\text { estrategias de } \\
\text { afrontamiento } \\
\text { que han desa- } \\
\text { rrollado dos } \\
\text { víctimas del } \\
\text { desplazamien- } \\
\text { to forzado del } \\
\text { corregimiento } \\
\text { de El Salado, } \\
\text { Bolívar y analizar } \\
\text { el impacto del } \\
\text { desplazamiento } \\
\text { en los ámbitos } \\
\text { familiar, social y } \\
\text { económico }\end{array}$} & \multirow{3}{*}{$\begin{array}{l}\text { Estrategia de } \\
\text { afrontamiento } \\
\text { cognitiva. }\end{array}$} & Planificación & $\begin{array}{l}\text { Estudiar la problemática, y } \\
\text { analizar las distintas opcio- } \\
\text { nes con el fin de desarrollar } \\
\text { técnicas para darle una so- } \\
\text { lución (Folkman y Lazarus, } \\
\text { 1988). }\end{array}$ \\
\hline & & Preocupación & $\begin{array}{l}\text { Hace referencia a una preo- } \\
\text { cupación improductiva por } \\
\text { el futuro, la cual, se relaciona } \\
\text { con afectividades negativas } \\
\text { como la angustia, la infeli- } \\
\text { cidad y el pesimismo (Folk- } \\
\text { man y Lazarus, 1988). }\end{array}$ \\
\hline & & $\begin{array}{l}\text { Reevaluación } \\
\text { positiva }\end{array}$ & $\begin{array}{l}\text { Hacer un cambio ante el sig- } \\
\text { nificado de la situación pro- } \\
\text { blema, resaltando aspectos } \\
\text { positivos que este tenga, ex- } \\
\text { presando optimismo y una } \\
\text { parte positiva de la situación } \\
\text { (Folkman y Lazarus, 1988). }\end{array}$ \\
\hline
\end{tabular}




\begin{tabular}{|c|c|c|c|}
\hline Objetivos & Categoría & subcategorías & Propiedades \\
\hline $\begin{array}{l}\text { Objetivo General } \\
\text { Describir las } \\
\text { estrategias de } \\
\text { afrontamiento } \\
\text { que han desa- } \\
\text { rrollado dos } \\
\text { víctimas del } \\
\text { desplazamien- } \\
\text { to forzado del }\end{array}$ & $\begin{array}{l}\text { Estrategia de } \\
\text { Afrontamiento } \\
\text { afectivo. }\end{array}$ & Apoyo social & $\begin{array}{l}\text { Implica asistir a otras perso- } \\
\text { nas cercanas como amigos o } \\
\text { familiares, organizaciones, } \\
\text { grupos, líderes espirituales } \\
\text { o profesionales, con el fin de } \\
\text { buscar ayuda material como } \\
\text { dinero, compartir la expe- } \\
\text { riencia con los demás, infor- } \\
\text { mación o apoyo emocional y } \\
\text { espiritual (Folkman y Laza- } \\
\text { rus, 1988). }\end{array}$ \\
\hline $\begin{array}{l}\text { de El Salado, } \\
\text { Bolívar y analizar } \\
\text { el impacto del } \\
\text { desplazamiento } \\
\text { en los ámbitos } \\
\text { familiar, social y } \\
\text { económico }\end{array}$ & $\begin{array}{l}\text { Estrategia de } \\
\text { afrontamiento } \\
\text { conductual }\end{array}$ & $\begin{array}{l}\text { Falta de afronta- } \\
\text { miento }\end{array}$ & $\begin{array}{l}\text { es la falta de capacidad por } \\
\text { parte del sujeto para afrontar } \\
\text { los problemas, dado a la falta } \\
\text { de dominio sobre sí mismo, } \\
\text { o al considerarlo irresoluble, } \\
\text { desarrollando síntomas psi- } \\
\text { cosomáticos y actitudes de- } \\
\text { presivas (Folkman y Lazarus, } \\
\text { 1988). }\end{array}$ \\
\hline
\end{tabular}

Fuente: Elaboración propia

\section{Categoría 1. Estrategia de afrontamiento cognitivo}

Después de haber analizado las entrevistas de los participantes, familiares y personas expertas encontramos que dentro de las estrategias de afrontamiento de tipo cognitivas mayormente utilizadas por las dos personas víctimas del desplazamiento está la preocupación, pues al vivenciar esta situación tienen pensamientos de angustia, infelicidad y pesimismo, como lo demuestra el participante $\mathrm{N}^{\circ} 2$ : "Pensé ya que las cosas ya no se podía, no era igual, ya el Salado no sería igual, y sería un pueblo fantasma". Asimismo, su familiar lo corrobora argumentado: "Yo lo noté a él muy preocupado, estresado".

De igual forma, al entrevistar a las personas expertas, también aseguran que una de las estrategias más utilizadas es la preocupación, como lo refiere la persona experta $\mathrm{N}^{\circ} 1$ : "Constante pesimismo, desesperanza, frustración, impotencia, sentimientos de angustia, de ansiedad y de desesperación constante en cada una de las situaciones que se les presente 
Estrategias de afrontamiento en víctimas desplazadas del corregimiento del Salado, Solívar. Un estudio de caso

de los hechos vividos", Y así, la experta No 2: "La gente nos narraba la historia con un nivel de desgarro, con un nivel de dolor y eso siempre lo he dicho, muchas víctimas narran con un inmenso dolor, es decir, tu evidencia que no existe ningún tipo de transformación, otras víctimas narran con una desesperanza, otros narraban con odio y vergüenza".

Cabe destacar que estas personas, aunque demostraron pensamientos de preocupación, también lograron hacer un cambio en la percepción que tenían sobre la problemática; este cambio fue positivo, pues demostraron un optimismo por salir adelante. Lo que indica que, de igual forma, las victimas utilizan la estrategia de afrontamiento cognitiva, reevaluación positiva. Como lo resalta en su discurso el participante $\mathrm{N}^{\circ}$ 1: "Salimos adelante con nuestros propios medios, pero con esa capacidad que teníamos de resolver nuestros propios problemas en ese momento". Convirtiéndose el estudio en una herramienta para poder salir adelante y obtener un mejor estilo de vida. Tal y como lo menciona la persona experta N²: "Porque pudieron crecer, pudieron estudiar, tener un nivel de vida un poco mejor". Con respecto a un familiar del participante, este recalca: "Él tuvo pensamientos de estudiar y fue, y hizo sus cursos ahí en el Sena”.

\section{Categoría 2. Estrategia de afrontamiento afectivo}

Con respecto a las estrategias de afrontamiento de tipo afectivo las dos personas víctimas del desplazamiento hacen uso del apoyo social, es decir, luego de vivir esta experiencia y ubicarse en otros lugares buscan ayuda de sus familiares o amigos cercanos, como también de organizaciones sociales que cuenten con apoyo profesional y espiritual, con el fin de obtener auxilios económicos y emocionales. Esto se denota en lo argumentado por el participante $\mathrm{N}^{\circ} 2$ : "Para recuperarme tuve que buscar ayuda psicológica, profesional". De igual forma, su familiar lo confirma diciendo: "Buscó ayuda psicológica." Y en la entrevista realizada a la persona experta $\mathrm{N}^{\circ} 2$ se evidenció: "Aproximadamente menos de la mitad contaron con el apoyo de una red de apoyo que podría ser grupo familiar, vecinos, organizaciones fundaciones y en algunos casos el Estado. 


\section{Categoría 3. Estrategia de afrontamiento conductual}

Luego de analizar las entrevistas se obtuvo que dentro de las estrategias de afrontamiento de tipo conductual, las dos personas víctimas solo utilizaron la falta de afrontamiento, es decir, no presentaron una capacidad para afrontar la situación, y por ende, desarrollaron síntomas psicosomáticos y depresivos, tal como lo refiere el participante №2: "A mí me cayó una autoestima, caí en ataque de pánico y tuve que ser sometido varios años con varias sesiones de psicología para poder superar mi trauma y todavía estoy medicado con la psiquiatría". Asimismo, su familiar lo reafirma argumentando: "Tuvo muchos problemas psicológicos porque todo el mundo no tiene la preparación para resistir ciertos impactos y eso lo afecto mucho".

\section{Discusión}

Teniendo en cuenta que el objetivo principal de esta investigación es describir las estrategias de afrontamiento que han desarrollado dos víctimas del desplazamiento forzado del corregimiento El Salado, Bolívar. A partir de entrevistas realizadas, se encontró que estas personas hacen uso de distintas estrategias de afrontamiento de tipo cognitiva, afectiva y conductual, las cuales son planteadas por Folkman y Lazarus (1988).

Cabe resaltar que de las estrategias mencionadas por estos autores se encuentran algunas de aspecto positivo y otras de aspecto negativo; en las personas entrevistadas se destaca un contraste, dado que uno de ellos demostró desarrollar estrategias positivas, de afrontamiento cognitivo como la reevaluación positiva, la cual Folkman y Lazarus (1988), definen como aquello que implica hacer un cambio ante el significado de la situación problema, resaltando aspectos positivos que este tenga, expresando optimismo y una parte positiva de la situación.

Una investigación realizada con mujeres desplazadas del Urabá también encontró que una de las estrategias más utilizadas fue la reevaluación positiva, puesto que entre ellas hicieron grupos de apoyo para ayudarse a sí mismas, es decir, buscaron superar la situación, sacar lo positivo y actuar con optimismo (Ayala, Rentería, Sánchez, 2015). Así mismo, se investigó sobre las estrategias utilizadas por victimas del desplazamiento en 
Estrategias de afrontamiento en víctimas desplazadas del corregimiento del Salado, Solívar. Un estudio de caso

Colombia y se obtuvo que la estrategia de afrontamiento de reevaluación positiva disminuye la presencia de síntomas psicosomáticos puesto que estas personas buscan aprender y darle un nuevo significado a la situación (Hewitt et al., 2016).

En cuanto al otro participante se encontró que este refiere, sobre todo, los aspectos negativos, es decir, desarrolla estrategias de afrontamiento de tipo cognitivo como la preocupación, que según Folkman y Lazarus (1988) hace referencia a una preocupación improductiva por el futuro, la cual, se relaciona con afectividades negativas como la angustia, la infelicidad y el pesimismo.

Esta persona manifestaba una estrategia de afrontamiento de tipo conductual como la falta de afrontamiento, definida por Folkman y Lazarus (1988) como la falta de capacidad por parte del sujeto para afrontar los problemas, debido a la falta de dominio sobre sí mismo, o al considerarlo irresoluble, desarrollando síntomas psicosomáticos y actitudes depresivas. Ante esto último, un estudio realizado en Colombia con personas víctimas del desplazamiento encontró que las personas que hacen uso de habilidades de afrontamiento como la falta de afrontamiento tienden a manifestar un aumento en la presencia de ansiedad - depresión (Hewitt et al., 2016).

Finalmente, en cuanto a las estrategias de afrontamiento de tipo afectivo, las victimas desarrollaron la de apoyo social. Según lo definen Folkman y Lazarus (1988), estos sujetos acudieron a otras personas cercanas como amigos o familiares, organizaciones, grupos, líderes espirituales o profesionales, con el fin de solicitar ayuda material como dinero, compartir la experiencia con los demás, algo de información o apoyo emocional y espiritual. Así mismo, una investigación realizada con familias desplazadas en Bogotá, señalan que gran parte de estas familias cuentan con familiares o conocidos que los acoge y viven un tiempo con ellos, logrando construir relaciones eficaces y nuevas redes afectivas (Gonzales, 2004).

En contraste a esto, el estudio realizado con estudiantes victimas del desplazamiento, adscritos al Programa de Psicología de la Corporación Universitaria del Caribe - CECAR, se encontró que estas personas no hicieron un uso del apoyo social; buscaron solucionar sus problemas de manera individual (Álvarez, Velilla \& Amell, 2018). 
Finalmente, cabe destacar que dentro de esta investigación se obtuvo una nueva estrategia de afrontamiento que ha sido utilizada por una de las victimas la cual es la basada en la Fe, pues añade que creer en Dios es una de las formas para salir adelante y mejorar la situación. En referencia a esto, un estudio realizado con adultos expuestos al conflicto armado en Colombia, indicó que estos hacen uso de la religión, debido a que es un factor de protección (Hewitt, et al., 2016). Otra investigación buscó describir las estrategias de afrontamiento utilizadas por hombres y mujeres desplazados encontrando que especialmente, las mujeres buscan apoyo en la religión pues creen que aporta aspectos positivos los cuales les fortalecen para afrontar la nueva situación (Aparicio, Di-colloredo y Moreno, 2007). De igual forma, un estudio realizado con desplazados internos en la ciudad de Bogotá, denotó que estas personas dicen que el creer en Dios les da fuerza para afrontar la situación por la cual pasaron y así seguir luchando (Zuluaga, 2016).

\section{Conclusiones}

Por medio de esta investigación se buscaba conocer los estilos de afrontamientos, frente a la experiencia del desplazamiento forzado en victimas del corregimiento El Salado, Bolívar. Luego de analizar las entrevistas semiestructuradas realizadas a dos víctimas, dos familiares y dos personas expertas en el tema se encontró que una de las estrategias cognitivas más utilizadas por ambas víctimas es la preocupación y esto se logró corroborar con las personas expertas. De igual forma, se constató que otra estrategia de tipo cognitivo es la reevaluación positiva, que implica hacer cambios en el significado del problema, resaltando aspectos positivos y expresando optimismo.

En las estrategias de tipo afectivo se encontró que ambas víctimas hacen uso del apoyo social buscando apoyo en familiares, amigos y profesionales que pudieran brindarles el soporte requerido. Por otra parte, en las estrategias de tipo conductual se obtuvo que una de las víctimas solo utilizó la falta de afrontamiento, por ende, está propenso a desarrollar síntomas psicosomáticos y depresivos como sucedió en este participante. 
Estrategias de afrontamiento en víctimas desplazadas del corregimiento del Salado, Solívar. Un estudio de caso

Cabe acotar que en esta investigación se encontró una nueva categoría la cual toma el nombre de estrategia basada en la Fe, ya que según ellos les permite tomar fuerzas para salir adelante después del desplazamiento.

\section{Referencias}

Álvarez, C., Velilla G., \& Amell, M., (2018). Estrategias de afrontamiento usadas por estudiantes víctimas del conflicto armado, vinculados al programa de Psicología de la Corporación Universitaria del Caribe - CECAR (Tesis de pregrado) Corporación Universitario del Caribe - CECAR. Sincelejo.

Aparicio, D., Di-colloredo, C., Moreno, J, (diciembre 2007). Descripción de los estilos de afrontamiento en hombres y mujeres ante la situación de desplazamiento. Psychologia. Avances de la disciplina, 1(2) Recuperado de http://www.redalyc.org/pdf/2972/297224996002.pdf.

Ávila, C. (2014). ATLAS. Ti como posibilidad para el docente investigador de representaciones sociales. ATLAS, TI QUALITIVE DATA ANALYSIS. Recuperado de https://atlasti.com/2014/11/17/atlas-ti-como-posibilidad-para-el-docente-investigador-de-representaciones-sociales/.

Ayala, L. M., Renteria, M. S., \& Sánchez, L. (2015). Estrategias de afrontamiento en un grupo de mujeres víctimas de violencia que hacen parte del colectivo el plantón en el municipio de Apartadó - Antioquia. (Trabajo de grado). Recuperado de http://200.24.17.74:8080/ jspui/bitstream/fcsh/321/1/AyalaLina_estrategiasafrontamientomujeresvictimasconflictoapartado.pdf.

Carver, C. S., Scheier, M. F., \& Weintraub, J. K. (1989). Assessing coping strategies: a theoretically based approach. Journal of personality and social psychology, 56(2), 267.

Compas, B. E., Davis, G. E., Forsythe, C. J., \& Wagner, B. M. (1987). Assessment of major and daily stressful events during adolescence: the Adolescent Perceived Events Scale. Journal of consulting and clinical psychology, 55(4), 534.

Folkman, S., \& Lazarus, R. S. (1988). Coping as a mediator of emotion. Journal of personality and social psychology, 54(3), 466. 
Galán, A. y Garcelán, S. (2001). Algunas aportaciones críticas en torno a la búsqueda de un marco teórico del afrontamiento en la psicosis. Psicothema, 13(4), 563-570.

Glaser, B. y Strauss, A. (1967) The Discovery of Grounded Theory. Strategies for Qualitative Research. New York. Aldine de Gruyter.

Gonzales, C. (2004). Transformación y resiliencia en familias desplazadas por la violencia hacia Bogotá. Revista de Estudios Sociales. (18) $123-$ 129. Bogotá. Recuperado de http://www.scielo.org.co/scielo.php?pi $\mathrm{d}=$ S0123-885X2004000200013\&script=sci_abstract\&tlng=es.

Hewitt, N., Juárez, F., Parad, A., Guerrero, J., Romero, Y., Salgado, A., Vargas, M. (2016). Afectaciones Psicológicas, Estrategias de Afrontamiento y Niveles de Resiliencia de Adultos Expuestos al Conflicto Armado en Colombia. Revista colombiana de psicología, 25 (1). Bogotá. Recuperado de http://www.scielo.org.co/pdf/rcps/v25nl/ v25nla09.pdf.

Martin, J. (2014). Estrategias de afrontamiento. Psicoterapia Online: todo es mente. Recuperado de http://todoesmente.com/defensa/estrategias-de-afrontamiento.

Martínez, J. (2011). Métodos de investigación cualitativa. Revista de la Corporación Internacional para el Desarrollo Educativo (8) 2930. Bogotá. Recuperado de http://www.cide.edu.co/doc/investigacion/3.\%20metodos\%20de\%20investigacion.pdf.

Monje, C. (2011). Metodología de la investigación en ciencias sociales. Metodología de la investigación cuantitativa y cualitativa: guía didáctica. Universidad Surcolombiana. Neiva. Recuperado de https://www.uv.mx/rmipe/files/2017/02/Guia-didactica-metodologia-de-la-investigacion.pdf.

Moos, R. H. \& Moos, B. S. (1988). Coping responses inventory manual.

Patton M. (2002). Qualitative research and evaluation methods. 3rd ed. Thousand Oaks: Sage. Recuperado de https://us.sagepub.com/enus/sam/qualitative-research-evaluation-methods/book232962.

Páez, D. (1993). Trauma político y memoria colectiva: Freud, Halbwachs y la psicología política contemporánea. Psicología política, (6), 7-34.

Rodríguez, M. (2006). Eficacia de un programa de grupo estructurado en estrategias de afrontamiento para dspt en adultos y adolescentes en 
Estrategias de afrontamiento en víctimas desplazadas del corregimiento del Salado, Solívar. Un estudio de caso

situación de desplazamiento. Universitas psychologica, 2(5), 3-10. Recuperado de http://www.scielo.org.co/scielo.php?script=sci_arttext\&pid=S1657-92672006000200005.

Sánchez, G. (2009). la masacre de El Salado: esa guerra no era nuestra. Recuperado de http://www.centrodememoriahistorica.gov.co/descargas/informes2009/informe_la_masacre_de_el_salado.pdf.

Vázquez, C. Crespo, M., \& Ring, J. (2000). Estrategias de afrontamiento. Medición clínica en psiquiatría y psicología, 425-435.

Zuluaga, L. C. (2016). Estrategias de afrontamiento en un grupo de desplazados internos en la ciudad de Bogotá. Revista de Psicología Universidad. 